\title{
TESTE DE VIDA SEQÜENCIAL COM DISTRIBUIÇÕES DE AMOSTRAGEM WEIBULL E WEIBULL INVERTIDA
}

\author{
Daniel I. De Souza Jr ${ }^{1}$.
}

Resumo: O mecanismo de teste de vida seqüencial representa uma situação de teste de hipótese na qual é tomada uma decisão de se aceitar ou se rejeitar uma hipótese nula, a qual se acredita ser a verdadeira, ou então se continuar amostrando. A distribuição Weibull Invertida tem sido utilizada em estimação Bayesiana representando a informação disponível sobre o parâmetro de forma de um modelo de amostragem Weibull (Erto [1]; De Souza e Lamberson [2]). Ela foi também empregada como a distribuição de amostragem de um teste de vida seqüencial por De Souza [3]. Tal como o modelo Weibull, possui dois parâmetros, um de forma e um de escala. O de localização é considerado ser igual à zero. Isso significa que o produto poderá vir a falhar no momento em que é colocado em uso. O modelo Weibull tem sido largamente utilizado como distribuição de falhas, principalmente para componentes mecânicos e metalúrgicos (De Souza 1997 [4], 1999 [5], 2000 [6] and 2001 [7]). Acontece que quando o seu parâmetro de forma for maior do que sete, a curva Weibull se torna acentuadamente em forma de pico, resultando com isso dificuldades computacionais (precisão) no cálculo dos valores da característica de interesse de um componente sendo testado. Em situações como essa, a distribuição Weibull Invertida parece fornecer uma melhor resposta ao problema de precisão apresentado pelo modelo Weibull. Nesse trabalho iremos analisar essa possibilidade e aplicaremos os mecanismos de teste de vida seqüencial derivados anteriormente por De Souza [3] e [6], nos quais as distribuições de amostragem foram, respectivamente, os modelos Weibull Invertido e Weibull. Um exemplo irá ilustrar a situação proposta.

Palavras-chave: Modelos Weibull e Weibull Invertido, Teste de Vida Seqüencial, Teste de Hipóteses, Parâmetros de Forma e de Escala.

Abstract: The sequential life testing approach is a hypothesis testing situation in which a decision is made about accepting, rejecting or continuing sampling as observations become available. The Inverse Weibull distribution was derived by Pascoal Erto [1]. It has been used in Bayesian reliability estimation to represent the information available about the shape parameter of an underlying Weibull sampling distribution (Erto [1]; De Souza \& Lamberson [2]). It has a location, a scale and a shape parameter. It has been also used before in a sequential life testing situation by De Souza [3]. The Weibull distribution is widely used as a failure model, particularly for metallurgical and mechanical components (De Souza 1997 [4], 1999 [5], 2000 [6] and 2001 [7]). It also has a location, a scale and a shape parameter. It happens that when its shape parameter value is greater than 7 , the Weibull curve becomes highly pointed, resulting in some computational difficulty (accuracy) in calculating the component's characteristic of interest values. In situations where the shape parameter values of the Weibull distribution are above 7, the Inverse Weibull distribution seems to have a better answer to the accuracy problem presented by the Weibull model. In this work we will analyze this possibility and we will apply sequential life testing approaches derived before by De Souza [7] and [6], in which the underlying sampling distributions were, respectively, the Inverse Weibull and Weibull models. An example will illustrate the application of a sequential life testing approach when the underlying sampling distributions are the Inverse Weibull and Weibull models.

Key-words: Weibull and Inverse Weibull models, Hypothesis Testing, Sequential Life Testing, Shape and Scale Parameters.

${ }^{1}$ UFF-TEC e UENF-Eng. de Produção. University of Florida, Industrial and Systems Engineering Dept. 


\section{INTRODUÇÃO}

A distribuição Weibull tem sido muito utilizada como modelo de falhas, principalmente para componentes mecânicos e metalúrgicos (De Souza [4], [5], [6] and [7]). Ela possui um parâmetro de forma e um de escala. O parâmetro de localização é considerado ser igual à zero. Isso significa que o produto poderá vir a falhar no momento em que é colocado em uso.

Acontece que quando o seu parâmetro de forma for maior do que sete, a curva Weibull se torna acentuadamente em forma de pico, resultando com isso dificuldades computacionais (precisão) no cálculo dos valores da característica de interesse de um componente sendo testado. Em situações nas quais os valores do seu parâmetro de forma forem superiores a sete, como no exemplo apresentado nesse trabalho, a distribuição Weibull Invertida parece fornecer uma melhor resposta ao problema de precisão apresentado pelo modelo Weibull.

A Figura (1) abaixo apresenta as curvas de confiabilidade para os modelos Weibull Invertido e Weibull em uma situação na qual os parâmetros de forma ( $\beta$ ) de ambas as distribuições possuem o mesmo valor de 8,8 e ambos os parâmetros de escala $(\theta)$ apresentam um valor de 450 horas.

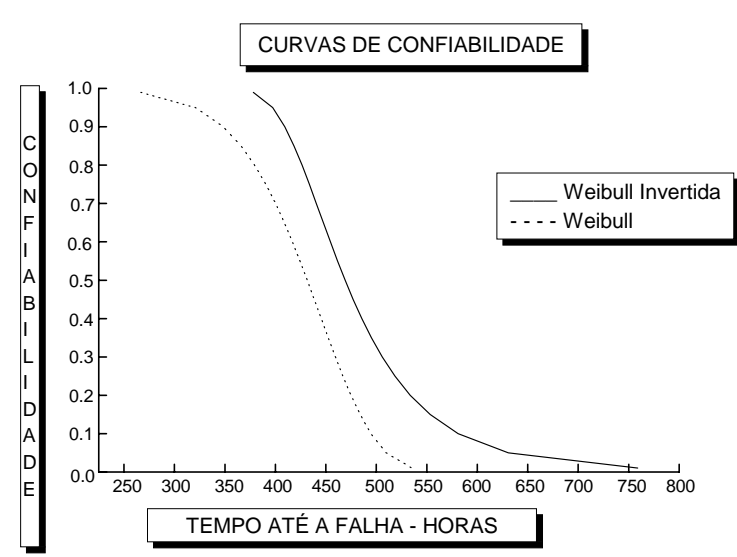

Figura 1. Curvas de confiabilidade para as distribuições Weibull Invertida e Weibull.
A função densidade Weibull é dada por

$$
\begin{aligned}
f(t) & =\frac{\beta}{\theta}\left(\frac{t}{\theta}\right)^{\beta-1} \exp \left[-\left(\frac{t}{\theta}\right)^{\beta}\right] \\
t & \geq 0 ; \quad \theta, \beta>0
\end{aligned}
$$

A distribuição Weibull Invertida tem sido utilizada em estimação Bayesiana representando a informação disponível sobre o parâmetro de forma de um modelo de amostragem Weibull (Erto [1]; De Souza e Lamberson [2]). Ela foi também empregada como a distribuição de amostragem de um teste de vida seqüencial por De Souza [3]. Possui dois parâmetros, um de forma e um de escala. Como no caso do modelo Weibull, o seu parâmetro de localização é considerado ser igual à zero. A sua função densidade é dada por

$$
\begin{gathered}
f(t)=\frac{\beta}{\theta}\left(\frac{\theta}{t}\right)^{\beta+1} \exp \left[-\left(\frac{\theta}{t}\right)^{\beta}\right] \\
t \geq 0 ; \theta, \beta>0
\end{gathered}
$$

As situações de teste de hipóteses serão dadas por

1. Para o parâmetro de escala $\theta$ :

$$
\mathrm{H}_{0}: \theta \geq \theta_{0} ; \quad \mathrm{H}_{1}: \theta<\theta_{0}
$$

A probabilidade de se aceitar $\mathrm{H}_{0}$ será dada por (1- $\alpha)$, no caso de $\theta=\theta_{0}$. Agora, caso $\theta=\theta_{1}$ onde $\theta_{1}<\theta_{0}$, então a probabilidade de se aceitar $\mathrm{H}_{0}$ terá um valor bem pequeno $\gamma$.

2. Para o parâmetro de forma $\beta$ :

$$
\mathrm{H}_{0}: \beta \geq \beta_{0} ; \quad \mathrm{H}_{1}: \beta<\beta_{0}
$$

A probabilidade de se aceitar $\mathrm{H}_{0}$ será novamente dada por (1- $\alpha)$, caso $\beta=\beta_{0}$. No caso de $\beta=\beta_{1}$ onde $\beta_{1}<\beta_{0}$, então a probabilidade de se aceitar $\mathrm{H}_{0}$ terá mais uma vez um valor bem pequeno $\gamma$. 


\section{TESTE SEQÜENCIAL}

Como já foi anteriormente mostrado por Kapur e Lamberson [8] e também por De Souza [6], o desenvolvimento de um teste de vida seqüencial utiliza a relação probabilística seqüencial (RPS). De acordo com De Souza [3], para o modelo Weibull Invertido de dois parâmetros, essa relação será dada por

$$
\begin{aligned}
\mathrm{RPS} & =\left(\frac{\beta_{1}}{\theta_{0}^{\beta_{0}}} \times \frac{\theta_{1}^{\beta_{1}}}{\beta_{0}}\right)^{\mathrm{n}} \prod_{\mathrm{i}=1}^{\mathrm{n}}\left(\mathrm{t}_{\mathrm{i}}\right) \beta_{0}-\beta_{1} \times \\
& \times \exp \left[-\sum_{\mathrm{i}=1}^{\mathrm{n}}\left(\begin{array}{cc}
\theta^{\beta_{1}} & \theta^{\beta_{0}} \\
\mathrm{t}_{\mathrm{i}}^{\beta_{1}} & -\frac{0_{0}}{\mathrm{t}_{0}} \\
\mathrm{i}
\end{array}\right)\right]
\end{aligned}
$$

A região contínua no teste é dada por $\mathrm{A}<\mathrm{SPR}<\mathrm{B}$, onde $\mathrm{A}=\gamma /(1-\alpha)$ e com $\mathrm{B}=(1-\gamma) / \alpha$. A hipótese nula $\mathrm{H}_{0}$ será aceita se $\mathrm{SPR} \geq \mathrm{B}$ e será rejeitada no caso de $\mathrm{SPR} \leq \mathrm{A}$. Agora, se $\mathrm{A}<\mathrm{SPR}<\mathrm{B}$, faremos mais uma observação. Logo, teremos

$$
\begin{aligned}
& \frac{\gamma}{(1-\alpha)}<\left(\frac{\beta_{1}}{\beta_{0}} \times \frac{\theta_{1}^{\beta_{1}}}{\beta_{0}}\right)^{\mathrm{n}} \prod_{\mathrm{i}=1}^{\mathrm{n}}\left(\mathrm{t}_{\mathrm{i}}\right) \beta_{0}-\beta_{1} \times \\
& \times \exp \left[-\sum_{\mathrm{i}=1}^{\mathrm{n}}\left(\frac{\theta^{\beta_{1}}}{\frac{1}{\mathrm{t}_{1}}}-\frac{\theta_{0}^{\beta_{0}}}{\mathrm{t}_{\mathrm{i}}^{\beta_{0}}}\right)\right]<\frac{(1-\gamma)}{\alpha}(3)
\end{aligned}
$$

Utilizando-se o logaritmo natural em cada um dos termos da equação acima, obteremos

$$
\begin{aligned}
& n \ln \left(\frac{\beta_{1}}{\theta_{0}^{\beta_{0}}} \times \frac{\theta_{1}^{\beta_{1}}}{\beta_{0}}\right)-\ln \left[\frac{(1-\gamma)}{\alpha}\right]<X< \\
& n \ln \left(\frac{\beta_{1}}{\theta_{0}^{\beta_{0}}} \times \frac{\theta_{1}^{\beta_{1}}}{\beta_{0}}\right)+\ln \left[\frac{(1-\alpha)}{\gamma}\right]
\end{aligned}
$$

$X=\sum_{i=1}^{n}\left(\frac{\theta_{1}^{\beta_{1}}}{t_{i}^{\beta_{1}}}-\frac{\theta_{0}^{\beta_{0}}}{t_{i}^{\beta_{0}}}\right)+\left(\beta_{1}-\beta_{0}\right) \sum_{i=1}^{n} \ln \left(t_{i}\right)(5)$

De acordo com De Souza [6], para o modelo Weibull, a relação probabilística seqüencial (RPS) será dada por

$$
\begin{aligned}
\operatorname{RPS} & =\left(\frac{\beta_{1}}{\beta_{1}} \times \frac{\theta_{0}^{\beta_{0}}}{\beta_{0}}\right)^{\mathrm{n}} \prod_{\mathrm{i}=1}^{\mathrm{n}}\left(\mathrm{t}_{\mathrm{i}}\right) \beta_{1}-\beta_{0} \times \\
& \times \exp \left[-\sum_{\mathrm{i}=1}^{\mathrm{n}}\left(\frac{\mathrm{t}_{\mathrm{i}}^{\beta_{1}}}{\theta^{\beta_{1}}}-\frac{\mathrm{t}_{\mathrm{i}}^{\beta_{0}}}{\theta_{0}^{\beta_{0}}}\right)\right]
\end{aligned}
$$

Novamente, a região contínua no teste é dada por $\mathrm{A}<\mathrm{SPR}<\mathrm{B}$, onde mais uma vez $A=\gamma /(1-\alpha)$ e $B=(1-\gamma) / \alpha$. A hipótese nula $\mathrm{H}_{0}$ será aceita se $\mathrm{SPR} \geq \mathrm{B}$ e será rejeitada no caso de $\mathrm{SPR} \leq \mathrm{A}$. Agora, se A $<$ SPR $<$ B, obteremos mais uma observação. Logo, teremos

$$
\begin{gathered}
\frac{\gamma}{(1-\alpha)}<\left(\frac{\beta_{1}}{\beta_{1}} \times \frac{\theta_{0}^{\beta_{0}}}{\beta_{0}}\right)^{\mathrm{n}} \prod_{\mathrm{i}=1}^{\mathrm{n}}\left(\mathrm{t}_{\mathrm{i}}\right) \beta_{1}-\beta_{0} \times \\
\times \exp \left[-\sum_{\mathrm{i}=1}^{\mathrm{n}}\left(\frac{\mathrm{t}_{\mathrm{i}}^{\beta_{1}}}{\theta_{1}^{\beta_{1}}}-\frac{\mathrm{t}^{\beta_{0}}}{\theta^{\beta_{0}}}\right)\right]<\frac{(1-\gamma)}{\alpha} \\
1
\end{gathered}
$$

Utilizando-se novamente o logaritmo natural em cada um dos termos da equação acima, obteremos

$$
\begin{gathered}
\operatorname{nln}\left(\frac{\beta_{1}}{\beta_{1}} \times \frac{\theta_{0}^{\beta_{0}}}{\beta_{0}}\right)-\ln \left[\frac{(1-\gamma)}{\alpha}\right]<X< \\
\operatorname{nln}\left(\frac{\beta_{1}}{\theta_{1}^{\beta_{1}}} \times \frac{\theta_{0}^{\beta_{0}}}{\beta_{0}}\right)+\ln \left[\frac{(1-\alpha)}{\gamma}\right]
\end{gathered}
$$


$X=\sum_{i=1}^{n}\left(\frac{t_{i}^{\beta_{1}}}{\theta_{1}^{\beta_{1}}}-\frac{t_{i}^{\beta_{0}}}{\theta_{0}^{\beta_{0}}}\right)+\left(\beta_{0}-\beta_{1}\right) \sum_{i=1}^{n} \ln \left(t_{i}\right)(7)$

\section{EXEMPLO}

Um produto eletrônico será submetido a um teste de vida seqüencial. Como esse é um produto bem conhecido, sabe-se que o mesmo poderá ser representado pelas distribuições de amostragem Weibull Invertida e Weibull, possuindo ambas valores para o parâmetro de escala $\theta$ de 420 horas e para o parâmetro de forma $\beta$ de 9,2. Escolheu-se para $\alpha$ o valor de 0,05 e para $\gamma$ o valor de 0,10. Esses valores de $\theta$ e de $\beta$ foram então selecionados como sendo os valores para os parâmetros da hipótese nula, ou seja, $\theta_{0}=420$ horas; $\beta_{0}=9,2$. Escolheu-se a seguir os valores de $\theta_{1}=490$ horas e de $\beta_{1}=8,4$ para os parâmetros da hipótese alternativa.

Logo, utilizando-se as equações (4) até a (7), teremos:

Para o modelo Weibull Invertido:

$$
\begin{aligned}
& n \ln \left(\frac{(490)^{8,4}}{(420)^{9,2}} \times \frac{8,4}{9,2}\right)-\ln \left[\frac{(1-0,10)}{0,05}\right]= \\
& =-3,6283 \times \mathrm{n}-2,890 \\
& n \ln \left(\frac{(490)^{8,4}}{(420)^{9,2}} \times \frac{8,4}{9,2}\right)+\ln \left[\frac{(1-0,05)}{0,10}\right]= \\
& =-3,6283 \times \mathrm{n}+2,251 \\
& X=\sum_{i=1}^{n}\left(\frac{(490)^{8,4}}{t_{i}^{8,4}}-\frac{(420)^{9,2}}{t_{i}^{9,2}}\right)+ \\
& +1,0 \times \sum_{i=1}^{n} \ln \left(t_{i}\right) . \log 0 \\
& -3,6283 \times \mathrm{n}-2,89<\mathrm{X}<-3,6283 \times \mathrm{n} \\
& +2,251
\end{aligned}
$$

Obtem-se agora uma amostra. O processo é então definido pelas seguintes regras:

1. Se $X \geq-3,6283 \times n+2,251$, aceitaremos $\mathrm{H}_{0}$.

2. Se $\mathrm{X} \leq-3,6283 \times \mathrm{n}-2,890$, rejeitaremos $\mathrm{H}_{0}$.

3. $\mathrm{Se}-3,6283 \times \mathrm{n}-2,890<\mathrm{X}<-$ $3,6283 \times \mathrm{n}+2,251$, faremos mais uma observação.

Nesse exemplo, foi necessário se testar apenas 6 itens para obtermos a decisão de aceitarmos a hipótese nula $\mathrm{H}_{0}$. Os tempos de falhas (horas) desses 6 itens foram: 467,02; 478,44; 412,20; 451,$10 ; 446,93$ e 433,05 horas. A Tabela (1) e a Figura (2) seguintes apresentam os resultados desse teste de vida.

Tabela 1 - Resultados do teste de vida seqüencial para a distribuição Weibull Invertida

\begin{tabular}{cccc}
\hline $\begin{array}{c}\text { Item } \\
\mathrm{N}^{\mathbf{0}}\end{array}$ & $\begin{array}{c}\text { Limite } \\
\text { Inferior }\end{array}$ & $\begin{array}{c}\text { Limite } \\
\text { Superior }\end{array}$ & $\begin{array}{c}\text { Valores } \\
\text { de X }\end{array}$ \\
\hline 1 & $-6,5183$ & $-1,3773$ & $-3,7968$ \\
2 & $-10,1466$ & $-5,0056$ & $-7,5936$ \\
3 & $-13,7749$ & $-8,6339$ & $-9,3260$ \\
4 & $-17,4032$ & $-12,2622$ & $-12,7303$ \\
5 & $-21,0315$ & $-15,8905$ & $-16,0109$ \\
6 & $-24,6598$ & $-19,5188$ & $-18,7991$ \\
\hline
\end{tabular}

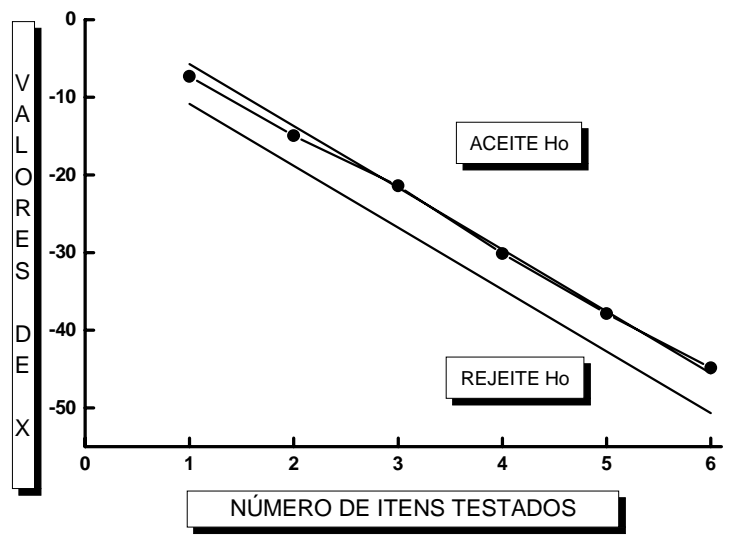

Figura 2. Resultados do teste de vida seqüencial para a distribuição Weibull Invertida.

Para o modelo Weibull:

$$
\operatorname{nln}\left(\frac{(420)^{9,2}}{(490)^{8,4}} \times \frac{8,4}{9,2}\right)-\ln \left[\frac{(1-0,10)}{0,05}\right]=
$$




$$
\begin{aligned}
& =3,4464 \times \mathrm{n}-2,890 \\
& \operatorname{nln}\left(\frac{(420)^{9,2}}{(490)^{8,4}} \times \frac{8,4}{9,2}\right)+\ln \left[\frac{(1-0,05)}{0,10}\right]= \\
& =3,4464 \times \mathrm{n}+2,251 \\
& X=\sum_{i=1}^{n}\left(\frac{\mathrm{t}_{\mathrm{i}}^{8,4}}{(490)^{8,4}}-\frac{\mathrm{t}_{\mathrm{i}}^{9,2}}{(420)^{9,2}}\right)+ \\
& \quad+(9,2-8,4) \sum_{i=1}^{n} \ln \left(\mathrm{t}_{\mathrm{i}}\right)
\end{aligned}
$$

Utilizando-se agora os 6 itens obtidos nesse exemplo, teremos

1. Se $X \geq 3,4464 \times \mathrm{n}+2,251$, aceitaremos $\mathrm{H}_{0}$.

2. Se $\mathrm{X} \leq 3,4464 \times \mathrm{n}-2,890$, rejeitaremos $\mathrm{H}_{0}$.

3. Se $3,4464 \times n-2,890<X<3,4464 \times$ $\mathrm{n}+2,251$, faremos novamente mais uma observação.

A Tabela (2) e a Figura (3) seguintes apresentam o resultado desse teste de vida.

Tabela 2 - Resultados do teste de vida seqüencial para a distribuição Weibull

\begin{tabular}{cccc}
\hline $\begin{array}{c}\text { Item } \\
\mathrm{N}^{\mathrm{o}}\end{array}$ & $\begin{array}{c}\text { Limite } \\
\text { Inferior }\end{array}$ & $\begin{array}{c}\text { Limite } \\
\text { Superior }\end{array}$ & $\begin{array}{c}\text { Valores } \\
\text { de X }\end{array}$ \\
\hline 1 & 0,5564 & 5,6974 & 2,9305 \\
2 & 4,0028 & 9,1438 & 5,3699 \\
3 & 7,4492 & 12,5902 & 9,5795 \\
4 & 10,8956 & 16,0366 & 13,0386 \\
5 & 14,3420 & 19,4830 & 16,6109 \\
6 & 17,7884 & 22,9294 & 20,4967 \\
\hline
\end{tabular}

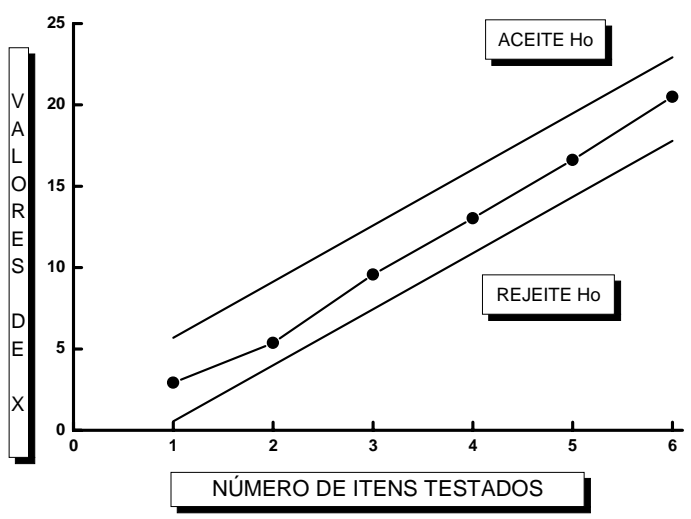

Figura 3. Resultados do teste de vida seqüencial para a distribuição Weibull.
Nesse caso, mesmo após a observação de 6 itens, não foi possível se chegar a uma decisão de se aceitar ou se rejeitar a hipótese nula $\mathrm{H}_{0}$ utilizando-se a distribuição de amostragem Weibull. À medida que o valor do seu parâmetro de forma $\beta$ aumenta $(\beta>7)$, a curva Weibull tende a uma forma de pico, resultando com isso dificuldades computacionais (precisão) no cálculo dos valores da característica de interesse de um componente sendo testado.

Nesse ponto, decidiu-se obter mais informações sobre o produto sendo testado através da realização de novas observações, até se atingir o ponto no qual seria possível se tomar uma decisão sobre a aceitação ou pela rejeição da hipótese nula $\mathrm{H}_{0}$. Tabela (3) e Figura (4) seguintes apresentam o resultado final desse teste de vida.

Tabela 3 - Resultado final do teste de vida seqüencial para a distribuição Weibull

\begin{tabular}{ccccc}
\hline $\begin{array}{c}\text { Item } \\
\mathbf{N}^{\mathbf{0}}\end{array}$ & $\begin{array}{c}\text { Limite } \\
\text { Inf. }\end{array}$ & $\begin{array}{c}\text { Limite } \\
\text { Sup. }\end{array}$ & $\begin{array}{c}\text { Valores } \\
\text { de X }\end{array}$ & $\begin{array}{c}\text { Tempo } \\
\text { De } \\
\text { Falhas } \\
\text { (Horas) }\end{array}$ \\
\hline 1 & 0,5564 & 5,6974 & 2,9305 & 467,02 \\
2 & 4,0028 & 9,1438 & 5,3699 & 478,44 \\
3 & 7,4492 & 12,590 & 9,5795 & 412,20 \\
4 & 10,896 & 16,037 & 13,039 & 451,10 \\
5 & 14,342 & 19,483 & 16,611 & 446,93 \\
6 & 17,788 & 22,929 & 20,497 & 433,05 \\
7 & 21,235 & 26,376 & 23,693 & 459,62 \\
8 & 24,681 & 29,822 & 27,767 & 422,16 \\
9 & 28,128 & 33,269 & 29,405 & 493,12 \\
10 & 31,574 & 36,715 & 33,143 & 440,06 \\
11 & 35,020 & 40,161 & 37,555 & 390,42 \\
12 & 38,467 & 43,608 & 42,122 & 332,02 \\
13 & 41,913 & 47,054 & 45,612 & 450,01 \\
14 & 45,360 & 50,500 & 49,819 & 412,36 \\
15 & 48,806 & 53,947 & 54,188 & 396,26 \\
\hline
\end{tabular}




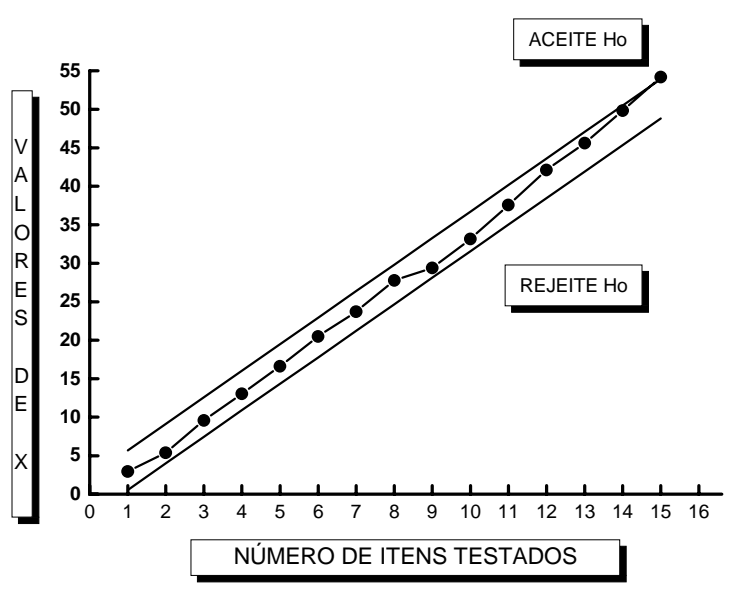

Figura 4. Resultado final do teste de vida seqüencial para a distribuição Weibull.

\section{CONCLUSÕES}

O mecanismo de teste de vida seqüencial desenvolvido nesse trabalho fornece regras para se trabalhar com a hipótese nula $\mathrm{H}_{0}$ em situações nas quais as distribuições de amostragem são os modelos Weibull Invertido e Weibull. Após a análise de cada observação, poderemos aceitar ou rejeitar a hipótese nula $\mathrm{H}_{0}$ ou ainda obter mais uma observação. No exemplo apresentado nesse artigo, quando a distribuição de amostragem era o modelo Weibull Invertido, foi necessário se analisar apenas 6 itens do produto eletrônico para se chegar a uma decisão de se aceitar a hipótese nula $\mathrm{H}_{0}$. Quando a distribuição de amostragem era o modelo Weibull, foi necessário se analisar 15 unidades do produto eletrônico para se obter a decisão de se aceitar a hipótese nula $\mathrm{H}_{0}$. À medida em que o valor do seu parâmetro de forma $\beta$ aumenta $(\beta>7)$, a curva Weibull se torna acentuadamente em forma de pico, resultando com isso dificuldades computacionais (precisão) no cálculo dos valores da característica de interesse de um componente sendo testado. Devido à concentração de densidade que se observa nessas situações de pico, qualquer aproximação no cálculo da característica de interesse poderá resultar em erros computacionais consideráveis. Isso sugere que para valores do parâmetro de forma $\beta$ superiores à 7,0 modelo Weibull Invertido deva ser utilizado como a distribuição de amostragem. Um exemplo ENGEVISTA, v. 6, n. 1, p. 28-34, abr. 2004 da aplicação dos modelos Weibull e Weibull Invertido de três parâmetros será apresentado em um próximo artigo. Devido ao número pequeno de observações necessárias para a sua utilização, o mecanismo de teste de vida seqüencial representa uma alternativa atraente ao da utilização de um teste de vida com um tamanho de amostra fixo, especialmente quando as distribuições de amostragem forem os modelos Weibull Invertido e Weibull.

\section{REFERÊNCIAS}

[1] Erto, Pasquale. 1982. "New Practical Bayes Estimators for the 2-Parameter Weibull Distribution", IEEE Transactions on Reliability, Vol. R-31, № 2, June 1982, pp. 194-197, USA.

[2] De Souza, Daniel I. \& Lamberson, Leonard R. 1995. Bayesian Weibull Reliability Estimation, IIE Transactions, 27(3):. 311-320; USA.

[3] De Souza, Daniel I. 2001. Truncation Mechanism in a Sequential Life Testing Approach with an Underlying TwoParameter Inverse Weibull Model, COMADEM 2001 Conference, Andrew G. Starr and Raj B. K. Rao (eds), Manchester, U.K., 4 - 6 September 2001, 809 - 816, Elsevier Science.

[4] De Souza, Daniel I. 1997. Costs Associated with Life Testing, Truncated After the Occurrence of a Predetermined Number $r$ of Failures. In Advances in Safety \& Reliability, Proceedings of the ESREL '97 International Conference on Safety and Reliability, Lisbon, Portugal, 17-20 June 1997, 3:1913-1920, New York: Elsevier Science.

[5] De Souza, Daniel I. 1999. Sequential life testing with an underlying Weibull sampling distribution. In Safety and Reliability, Schuëller \& Kafka eds., Proceedings of the ESREL '99 International Conference on Safety and Reliability, Garching, Germany, 13-17 September 1999, 2:869-874, Rotterdam,: Balkema.

[6] De Souza, Daniel I. 2000. Further Thoughts on a Sequential Life Testing Approach Using a Weibull Model, Foresight and Precaution, ESREL 2000 
Conference, Cottam, Harvey, Pape \& Tait (eds), Edinburgh; Scotland; 14-17 May 2000; 2: 1641 - 1647, Rotterdam,: Balkema.

[7] De Souza, Daniel I. 2001. Sequential Life Testing with a Truncation Mechanism for an Underlying Weibull Model, Towards a Safer World, ESREL 2001 Conference, Zio, Demichela \& Piccinini (eds), Torino, Italy, $16-20$ September 2001; 3: 1539 - 1546, Politecnico Di Torino.

[8] Kapur, Kailash \& Lamberson, Leonard R. 1977. Reliability in Engineering Design. New York: John Willey \& Sons, Inc. 